\title{
AIR POLLUTION RISK ASSESSMENT OF HAZARDOUS AND NOXIOUS SUBSTANCE-SPILL ACCIDENTS: A CASE STUDY OF ULSAN PORT, KOREA
}

\author{
NAMGYUN KIM \& HEEKYUNG PARK \\ Department of Civil and Environmental Engineering, KAIST, Republic of Korea
}

\begin{abstract}
The definition of Hazardous and Noxious Substances (HNS) has been defined by the Protocol on Preparedness, Response and Co-operation to pollution Incidents by Hazardous and Noxious Substances, 2000 (OPRC-HNS Protocol). HNS can be delivered both by land and sea through international trade, but are mainly traded over the seas. Europe, North America and Eastern Asia are the main trading regions of large quantities of HNS through shipping. HNS marine trade volume in Korea is about 251 million tons, accounting for $19 \%$ of total marine trade volume. Despite the rapid growth in transport of HNS by vessel, there is a lack of HNS-specialized response systems compared to oil response systems. In this study, a risk analysis was conducted of responses to HNS spill accidents, by analysing accident cases of Hanyang Ace in Ulsan port, Korea. In addition, based on the HNS spill accident scenario, the top three most concerned substances of HNS by the Korean government were selected, and the risk assessment was performed by using the attributes of the accident scenario in terms of sea state, weather condition and accident type. From the results, we found that there were no impacts of HNS spill accidents to the residents who live away from Ulsan port, but they showed more impact to the residents who live nearby Ulsan industrial area. Since atmospheric diffusion of HNS is invisible to bare eyes, it is necessary to prepare for HNS spill accidents, as shown by the risk assessment.

Keywords: HNS, risk assessment, scenario.
\end{abstract}

\section{INTRODUCTION}

According to the Protocol on Preparedness, Response and Co-operation to pollution Incidents by Hazardous and Noxious Substances proposed by International Maritime Organization, 2000 (IMO OPRC-HNS Protocol 2000) [1], the definition of Hazardous and Noxious Substances (HNS) has been defined that "any substance other than oil which, if introduced into the marine environment, is likely to create hazards to human health, to harm living resources and marine life, to damage amenities or to interfere with other legitimate uses of the sea.” HNS are mainly traded in large quantities through shipping oversea. Moreover, the main HNS exporters and importers are Europe, North America and Eastern Asia. In Korea, the maritime transport of HNS has been increased around 65\% in the last decades. It is more than 2.5 times higher that of the world HNS trade average.

Considering the increase trading volume of HNS in Korea, the risk of environmental pollution and human health are also though to increase due to HNS spill accidents. Table 1 shows the number of maritime pollution accidents and the total spill volume of oil and HNS from 2011 to 2015, respectively [2]. The average numbers of maritime pollution accidents are about 250 cases/year, and it is mainly occurred due to oil spill. The number of maritime pollution caused by HNS is about 3 cases/year on average, which is $1 \%$ of the total number of maritime pollution accidents. However, the average HNS spill volume is $56.4 \mathrm{kl}$, accounting for about $10 \%$ of the total spill volume. These outcomes show that a large amount of HNS is leaked to cause pollution while an HNS spill accident occurred.

In order to respond to the HNS spill accident, many studies are focused on HNS risk assessment. Neuparth et al. [3] developed a weight-of-evidence approach for an effective response to HNS spill that can control the environmental risk. Fuhrer et al. [4] compared the 
experiment results of styrene spillage in the environment with theoretical assessments. Garcia et al. [5] studied the risk associated with a potential HNS spill along the Italian coastline using comparative index. Lee and Jung [6] studied the risk assessment and national measure plan for oil and HNS spill accidents near Korea with previous accident data. Cho et al. [7] analysed the potential risk of HNS spill accidents by using event tree analysis.

The risk assessment of maritime HNS spill accident was mostly studied based on a statistical method of past accident cases. In addition, the risk of environmental pollution was mainly studied. In this study, the risk analysis was performed based on the environment and human risk according to the behaviour of HNS. Dynamic model was used to analysis risk area for Ulsan Port in Korea. Furthermore, the scenarios were based on the HNS spill accident of the Hanyang Ace in 2015.

\section{FIELD STUDY}

\subsection{Study area}

The study area is Ulsan Port in Maeam-dong, Nam-gu, Ulsan City, Korea. Fig. 1 shows the location of Ulsan Port. The industrial complex is built with Ulsan Port as its centre. A residential area is formed far from about $10 \mathrm{~km}$ northwest of the Port centre. In addition, east area from the centre port is mountainous area.

Table 1: The number of accidents and outflow volume.

\begin{tabular}{|l|c|c|c|c|c|}
\hline \multicolumn{1}{|c|}{ Year } & 2011 & 2012 & 2013 & 2014 & 2015 \\
\hline $\begin{array}{l}\text { The number of maritime } \\
\text { pollution accident }\end{array}$ & 287 & 253 & 252 & 215 & 250 \\
\hline The number of HNS accident & 4 & 2 & 4 & 3 & 3 \\
\hline The total spill volume (kl) & 369.1 & 418.7 & 265.0 & 2001.4 & 464.1 \\
\hline The HNS spill volume (kl) & 75.6 & 0.7 & 3.1 & 4.4 & 198 \\
\hline
\end{tabular}

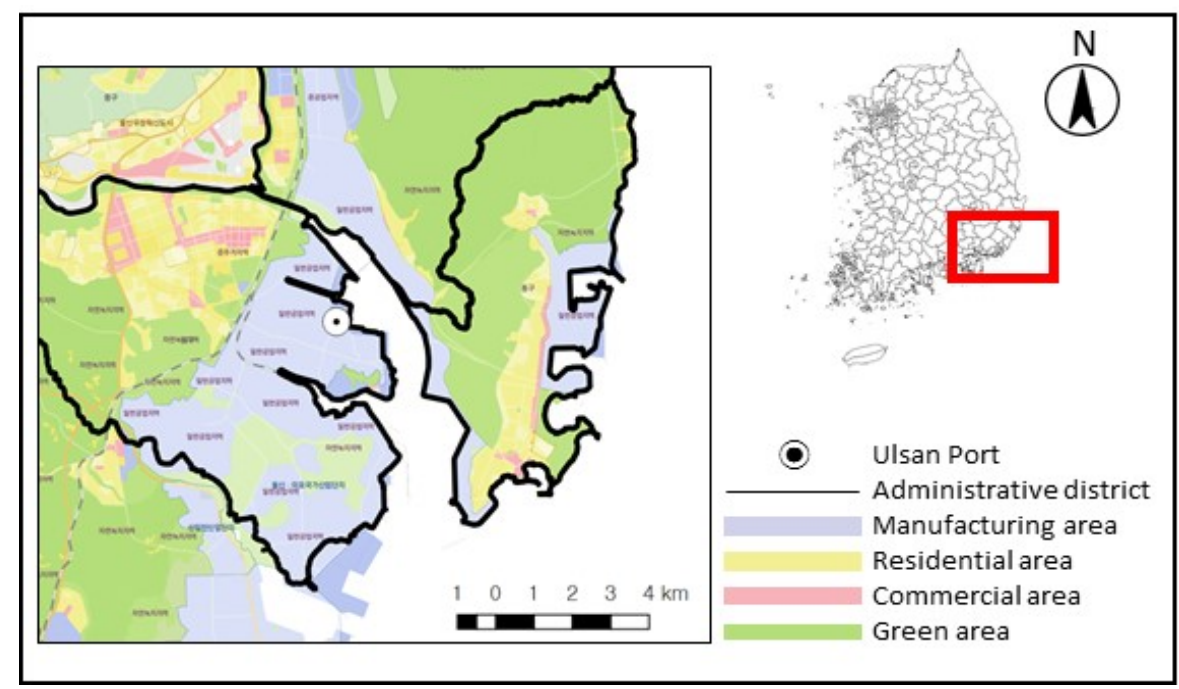

Figure 1: The location of Ulsan Port, Korea. 
Ulsan Port is an industrial port since 1963, and it has been developed with focusing on a port of heavy chemical industry. Ulsan Port is the third largest port of number of ship entering and clearing in Korea. The pollutant spill in Ulsan Port is mostly HNS. Based on Fig. 2, Ulsan Port showed that $4^{\text {th }}$ highest average volume of pollutant spill among all port in Korea, but also showed that the highest volume of pollutant spill during year of 2015. The volume of pollutant spill showed the highest in 2015 because of the accident of Hanyang Ace.

\subsection{HNS spill accident of the Hanyang Ace}

On January 11, 2015, explosion and fire occurred in cargo tanks during the shipment of Hanyang Ace. Hanyang Ace was a chemical vessel that contains mixed acid of $80 \%$ nitric acid and 20\% sulphuric acid. Due to the accident, four out of fifteen crew members were injured, and the mixed acid were spilled on the coast line of Ulsan port. According to a survey by Korean Coast Guard [8], the accident was occurred that mixed acid was leaked due to a small unknown crack at cargo tank and the explosion occurred due to the reaction between mixed acid and seawater in the ballast tank. Therefore, a pore was formed into the upper part of the cargo tank, and about $190 \mathrm{kl}$ of the mixed acid gas were spilled into the air (Fig. 3).

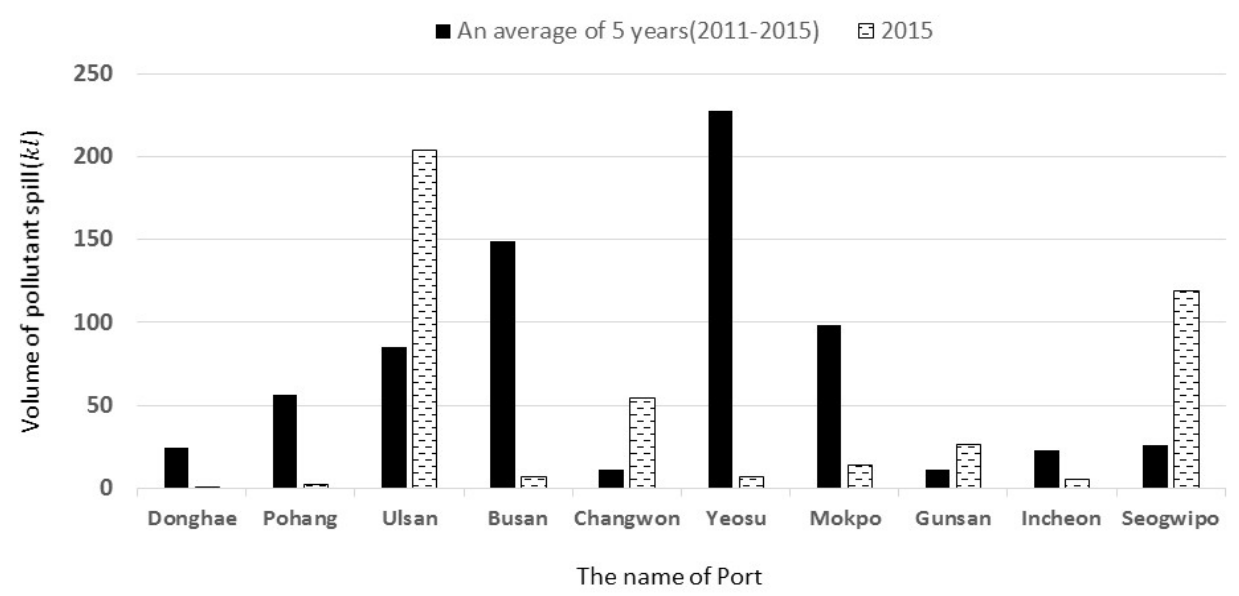

Figure 2: Volume of pollutant spill.
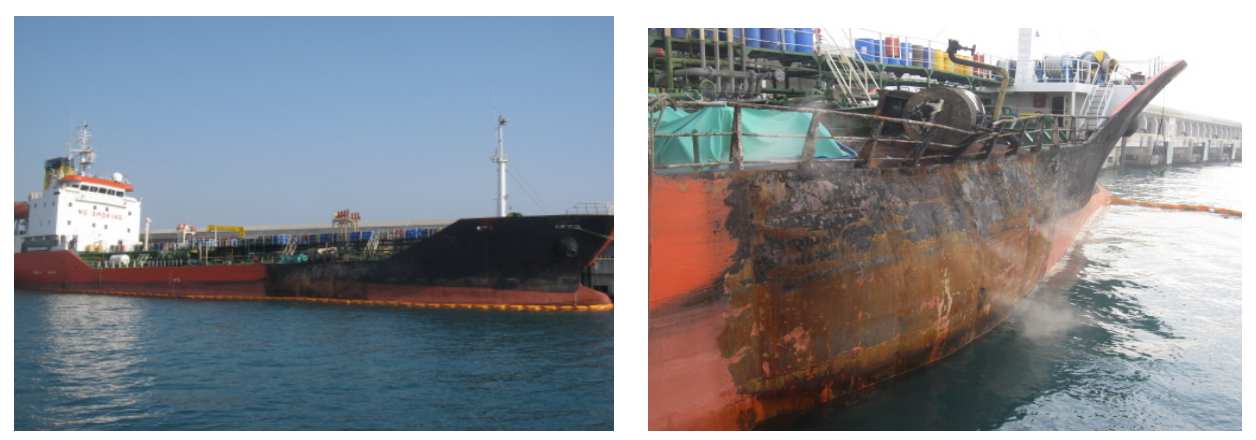

Figure 3: After the accident of Hanyang Ace [8]. 


\section{NUMERICAL SIMULATION}

\subsection{Background}

Areal Locations of Hazardous Atmospheres (ALOHA) was used for simulating the HNS spill accident on Ulsan Port. ALOHA allowed to estimate the HNS air dispersion after chemical spill. In addition, it can simulate not only HNS spill but also fire and explosion scenarios. ALOHA was composed of source strength model, air dispersion model, flammable area model and model for calculating blast effects from vapour cloud explosions. In this study, we adapted two models from ALOHA; source strength model and air dispersion model. Source strength model was applied to calculate the chemicals released from the tank and air dispersion model was applied to calculate volume of released buoyant gas and heavy gas in the air.

3.1.1 Internal temperature and pressure of the tank

The internal pressure in the tank is defined by the tank temperature and the internal temperature in the tank is determined as follows:

$$
\frac{d T_{T}}{d t}=\frac{Q_{e} L_{c}+F_{H w} A_{t w}}{\rho_{l} c_{p l} V_{l}}
$$

where $F_{H w}$ is the thermal energy flux across the walls, $A_{t w}$ dis the area of the tank walls in contact with liquid, $\rho_{l}$ is the density, $c_{p l}$ is heat capacity, $V_{l}$ is volume of the liquid within the tank, and $Q_{e}$ is the mass evaporation rate that is calculated by the following equation:

$$
Q_{e}(t)=\frac{Q_{T}}{\rho_{X}}\left(\frac{\rho_{l} \rho_{g}}{\rho_{l}-\rho_{g}}\right),
$$

where $Q_{T}$ is the total mass loss from the tank, $\rho_{X}$ is the density of the effluent fluid, and $\rho_{g}$ is the density of the gas.

3.1.2 Release of a 2-phase mixture of a liquid above its ambient boiling point HNS spill could be simulated a case of a superheated liquid and a 2-phase mixture from the flashing boiling of a superheated liquid [9], [10]. The equation of mass flux, $G$ is given as follows:

$$
G=\frac{L_{c}}{\left(v_{g}-v_{l}\right)}\left(\frac{1}{\sqrt{N c_{p} T}}\right)
$$

where, $L_{c}$ is the specific latent heat of vaporization, $v_{g}$ is the specific volume of the vapor, $v_{l}$ is the specific volume of the liquid, $c_{p}$ is the heat capacity of the fluid, and $T$ is the temperature.

\subsubsection{Dispersion model for buoyant gases}

This model is used to predict the concentration distribution. The longer the average time of measurement, the more likely it is inclined to the distribution to be in a Gaussian shape. This Gaussian model is modelled as a single one-hour steady-state spill which gives rise to a single cloud. This model developed by Palazzi [11] that described as follows:

$$
C(x, y, z, t)=\left\{\begin{array}{c}
\frac{\chi}{2}\left[\operatorname{erf}\left(\frac{x}{\sigma_{x} \sqrt{2}}\right)-\operatorname{erf}\left(\frac{x-U t}{\sigma_{x} \sqrt{2}}\right)\right],\left(t \leq t_{r}\right) \\
\frac{\chi}{2}\left[\operatorname{erf}\left(\frac{x-U\left(t-t_{r}\right)}{\sigma_{x} \sqrt{2}}\right)-\operatorname{erf}\left(\frac{x-U t}{\sigma_{x} \sqrt{2}}\right)\right],\left(t_{r}<t<\infty\right)
\end{array},\right.
$$




$$
\chi(x, y, z)=\frac{Q(t)}{U} g_{y}(x, y) g_{z}(x, z)
$$

where, $\sigma_{x}, \sigma_{y}, \sigma_{z}$ are dispersion parameters, $t_{r}$ is the duration of the spill, $U$ is wind speed, $\chi$ is Gaussian distribution from a continuous steady-state point source [12].

\subsubsection{Dispersion model for heavy gases}

The heavy gas dispersion model is based on the Dense Gas Dispersion (DEGADIS) model. DEGADIS model was developed by Colenbrander and Puttock [13]. The following equation shows the concentration of the pollutant:

$$
C(x, y, z)=\left\{\begin{array}{c}
c_{c}(x) \exp \left(-\frac{|y|-b(x)}{S_{y}(x)}\right)^{2}-\left(\frac{z}{S_{z}}\right)^{1+n},(|y|>b(x)) \\
c_{c}(x) \exp \left(-\left(\frac{z}{S_{z}}\right)^{1+n}\right),(|y| \leq b(x))
\end{array},\right.
$$

where, $c_{c}(x)$ is the centreline ground-level concentration, $S_{y}(x)$ is the lateral dispersion parameter, $S_{Z}(x)$ is vertical dispersion parameter, $b$ is the half-width of the homogeneous core section.

\subsection{Numerical simulation of the HNS spill accident of the Hanyang Ace}

The accident of Hanyang Ace was analysed by numerical modelling. To simulate the model, it is mainly needed location information, HNS information, and weather information. At the time of Hanyang Ace accident, temperature was $9.7^{\circ} \mathrm{C}$, wind speed was $5 \mathrm{~m} / \mathrm{s}$, wind direction was WNW, humidity was $22 \%$, and there was no cloudy weather. As mentioned above, the chemical substances are mixed acid with $80 \%$ nitric acid and $20 \%$ sulphuric acid, and the specific gravity of mixed acid is 1.6. The mixed acid was stored in the tank in a liquid state of about 300 tons, and the mixed acid to be spilled out due to a hole about $10 \mathrm{~cm}$ after a fire occurred.

Table 2 showed a numerical simulation results and field survey results. In the field data, about $320 \mathrm{~kg}$ of mixed acid was spilled, and a portion of the vessel was burned around $8 \mathrm{~m}$ in radius. The gas spilt out into the air was dispersed about $300 \mathrm{~m}$ away. As a result of the numerical simulation, the spilled gas was $68 \mathrm{~kg}$, and the spilled liquid was $396 \mathrm{~kg}$, respectively. The gas was dispersed about $650 \mathrm{~m}$, and fire range was $11 \mathrm{~m}$ in radius. Fig. 4 showed the scene of mixed acid gas spill and the fire. Fig. 5 showed the discharge of evaporation rate and burn rate, respectively. It was simulated that the gas was discharged at a rate of $25 \mathrm{~g} / \mathrm{min}$, and the burn rate was about $183 \mathrm{~g} / \mathrm{min}$. The numerical simulation results were overestimated. This was because the sizes of the simulated and actual hole from the accident were different. The actual hole was an $\mathrm{L}$ shape, and the length was about $10 \mathrm{~cm}$. However, the simulated hole was circular shape, and diameter was $10 \mathrm{~cm}$.

Table 2: Simulation results.

\begin{tabular}{|l|c|c|c|}
\hline \multirow{2}{*}{} & \multirow{2}{*}{ Actual data } & \multicolumn{2}{|c|}{ Simulation data } \\
\cline { 3 - 4 } & & gas & liquid \\
\hline Spilled volume (kg) & 320 & 68 & 396 \\
\hline Fire range (m (radius)) & 8 & - & 11 \\
\hline Dispersion distance (m) & 300 & 650 & - \\
\hline
\end{tabular}



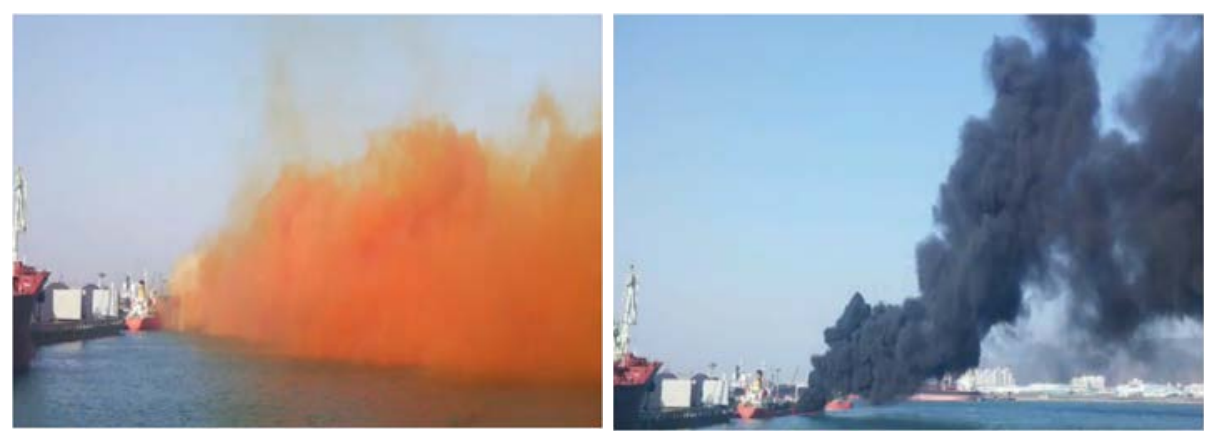

Figure 4: The scene of gas spill (left), and fire (right) [8].
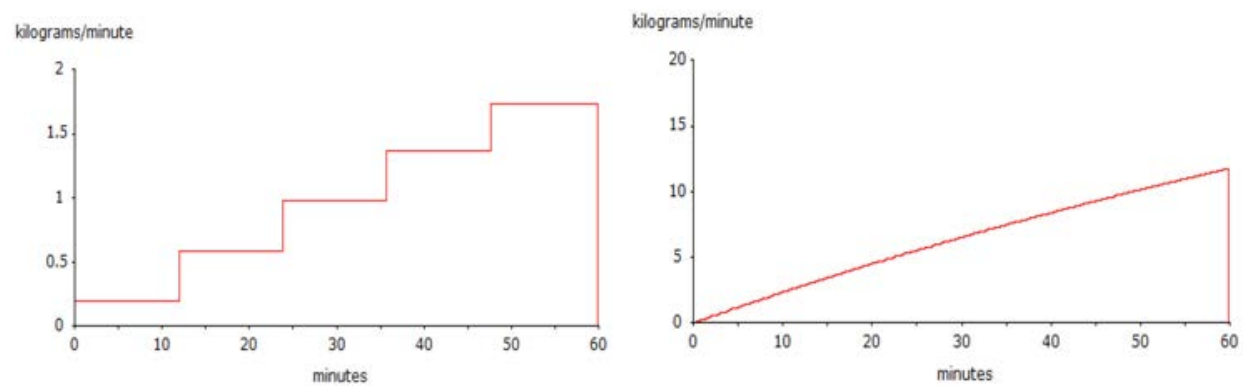

Figure 5: Numerical result of gas spill (left), and fire (right).

\section{RISK ASSESSMENT}

\subsection{Scenario of HNS spill accident on Ulsan Port}

In order to establish an accident scenario, it needed to consider the types of chemical substance and weather conditions. Based on this, chemical substances were selected: benzene, styrene, and propylene. These substances are traded more than 1,000tons/year among HNS traded at Ulan port. This value is the least amounts of trading volume for assessing the risk of HNS that compromised by European waters [14].

There are three types of accidents in HNS: spill, fire, and explosion. From among these, only spill accident was considered to the scenario, and it was based on the past HNS accident in Ulsan Port.

The accident scenario was considered with 6 different aspects; wind speed, accident type, temperature, humidity, tank size, and crack diameter. The wind speed was decided based on the class of Beaufort; wind class 1 (light air), class 4 (moderate breeze), and class 5 (gale), because the spread of gas type HNS are mainly influenced by the wind. The scenario was also considered of $17^{\circ} \mathrm{C}$ of temperature, $67 \%$ of humidity, $1012.3 \mathrm{hpa}$ of sea-level pressure. These values are the average environmental condition of Ulsan Port from 1980 to 2010. The size of HNS tank was set as that of Hanyang Ace accident, and the crack diameter of the hole was $20 \mathrm{~cm}$ (Table 3). 
Table 4 showed the risk dispersion distance due to HNS spill. The maximum risk dispersion distances were $2300 \mathrm{~m}$ (benzene), $913 \mathrm{~m}$ (styrene), and 2700m (propylene), respectively. The maximum risk dispersion distances were derived when wind speed was $1 \mathrm{~m} / \mathrm{s}$, and stronger the wind, shorter the risk distance. This is because the HNS was rapidly dispersed by the wind and concentration was lowered (Fig. 6).

The values of Table 4 were averaged to show the risk assessment map (Fig. 7). The radius of the AEGLs-1(red zone) stage was $320 \mathrm{~m}$, the radius of AEGLs-2 stage was $739 \mathrm{~m}$, and the radius of AEGL-3 stage was $1679 \mathrm{~m}$. From the result, it was confirmed that there was no negative effect to the residential area in Ulsan but health risk of the industrial area was increased due to HNS spill accidents. This result shows that the co-workers who work for those industrial areas in are needed to be evacuated. However, the risk range could be arranged by adding more of scenarios. Thus, it is necessary to add more scenarios to establish more reasonable risk map.

\subsection{Risk assessment of HNS spill accident on Ulsan Port}

The risk assessment of HNS spill accident in Ulsan Port was evaluated through numerical simulation based on the scenario. Risk assessment criteria were presented in accordance with acute exposure level guidelines (AEGLs). AEGLs were expressed as specific concentrations of airborne chemicals at which health effects that may occur. AEGLs assigned 1, 2, or 3 according to severity of effects, and each HNS has different criteria. AEGL-1 means that notable discomfort, irritation, or certain asymptomatic of non-sensory effects. However, the effects are not disabling and are transient and reversible upon cessation of exposure. AEGL2 means that irreversible or other serious, long-lasting adverse health effects or an impaired ability to escape. AEGL-3 means that life-threatening health effects or death. The exposure standard time is 1 hours. The concentration criteria of AEGL-1 are 52ppm (benzene), 20ppm (styrene) and 1500ppm (propylene). The criteria concentrations of AEGL-2 are 800ppm (benzene), 130ppm (styrene) and 2800ppm (propylene). The criteria concentrations of AEGL-3 are 4000ppm (benzene), 1100ppm (styrene) and 17000ppm (propylene).

Table 3: Accident scenario.

\begin{tabular}{|c|c|c|c|c|c|c|}
\hline HNS & $\begin{array}{c}\text { Wind speed } \\
\text { (Beaufort } \\
\text { number) }\end{array}$ & $\begin{array}{l}\text { Accident } \\
\text { type }\end{array}$ & Temperature & Humidity & $\begin{array}{l}\text { Tank } \\
\text { size }\end{array}$ & $\begin{array}{c}\text { Crack } \\
\text { diameter }\end{array}$ \\
\hline Benzene & $1(1 \mathrm{~m} / \mathrm{s})$ & \multirow{3}{*}{ Spill } & \multirow{3}{*}{$17\left({ }^{\circ} \mathrm{C}\right)$} & \multirow{3}{*}{ 67(\%) } & \multirow{3}{*}{$\begin{array}{l}188 \mathrm{~m}^{3} \\
(100 \%)\end{array}$} & \multirow{3}{*}{$20(\mathrm{~cm})$} \\
\hline Styrene & $4(6.5 \mathrm{~m} / \mathrm{s})$ & & & & & \\
\hline Propylene & $7(15 \mathrm{~m} / \mathrm{s})$ & & & & & \\
\hline
\end{tabular}

Table 4: Risk dispersion distance.

\begin{tabular}{|c|c|c|c|c|}
\hline \multicolumn{2}{|c|}{} & \multicolumn{3}{c|}{ Wind speed } \\
\cline { 3 - 5 } \multicolumn{2}{|c|}{} & $1 \mathrm{~m} / \mathrm{s}$ & $6.5 \mathrm{~m} / \mathrm{s}$ & $15 \mathrm{~m} / \mathrm{s}$ \\
\hline \multirow{3}{*}{ Benzene } & AEGL-1 & 258 & 75 & 63 \\
\cline { 2 - 5 } & AEGL-2 & 597 & 178 & 125 \\
\cline { 2 - 5 } & AEGL-3 & 2300 & 2000 & 1400 \\
\hline \multirow{3}{*}{ Styrene } & AEGL-1 & 200 & 98 & 96 \\
\cline { 2 - 5 } & AEGL-2 & 249 & 143 & 129 \\
\cline { 2 - 5 } & AEGL-3 & 913 & 711 & 590 \\
\hline \multirow{3}{*}{ Propylene } & AEGL-1 & 863 & 732 & 495 \\
\cline { 2 - 5 } & AEGL-2 & 2000 & 1700 & 2200 \\
\cline { 2 - 5 } & AEGL-3 & 2700 & 2300 & \\
\hline
\end{tabular}




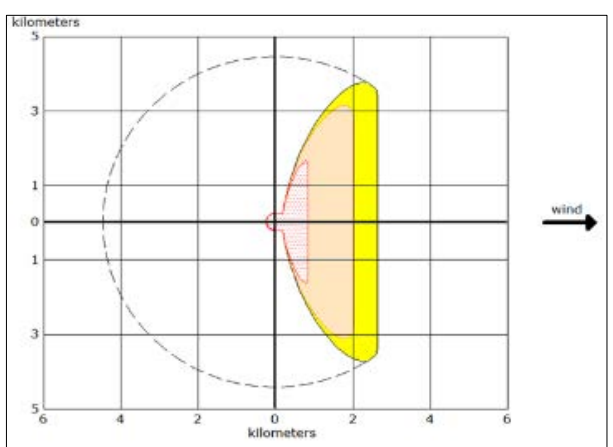

Wind speed: $1 \mathrm{~m} / \mathrm{s}$

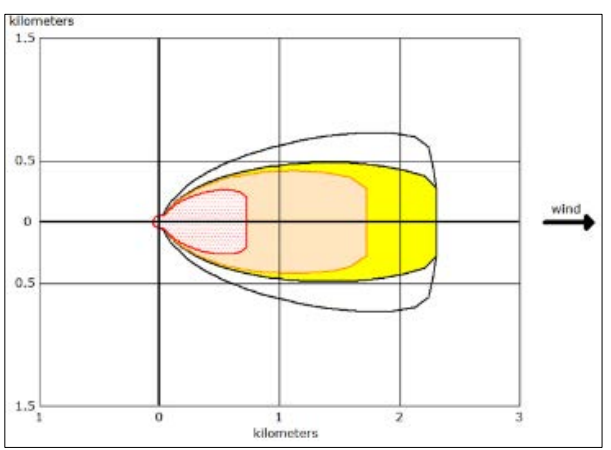

Wind speed: $6.5 \mathrm{~m} / \mathrm{s}$

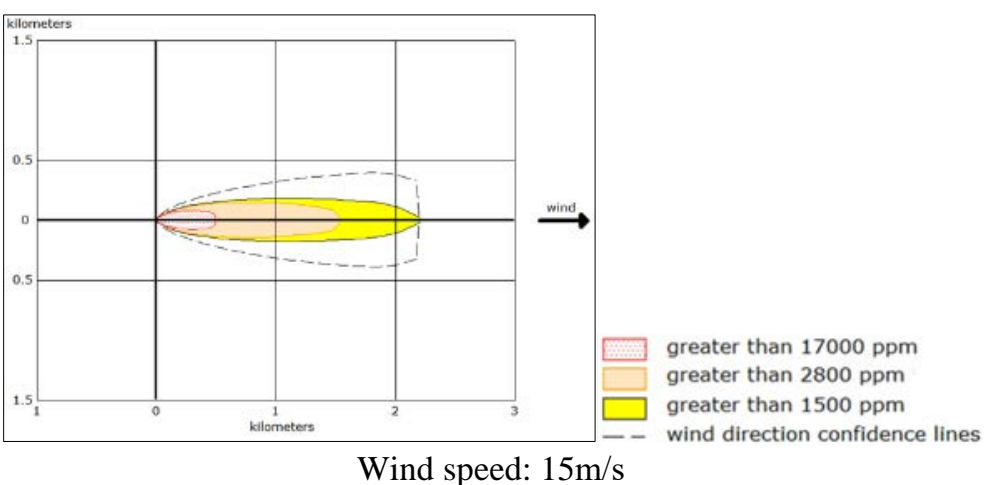

Figure 6: Risk dispersion area according to wind speed in propylene case.

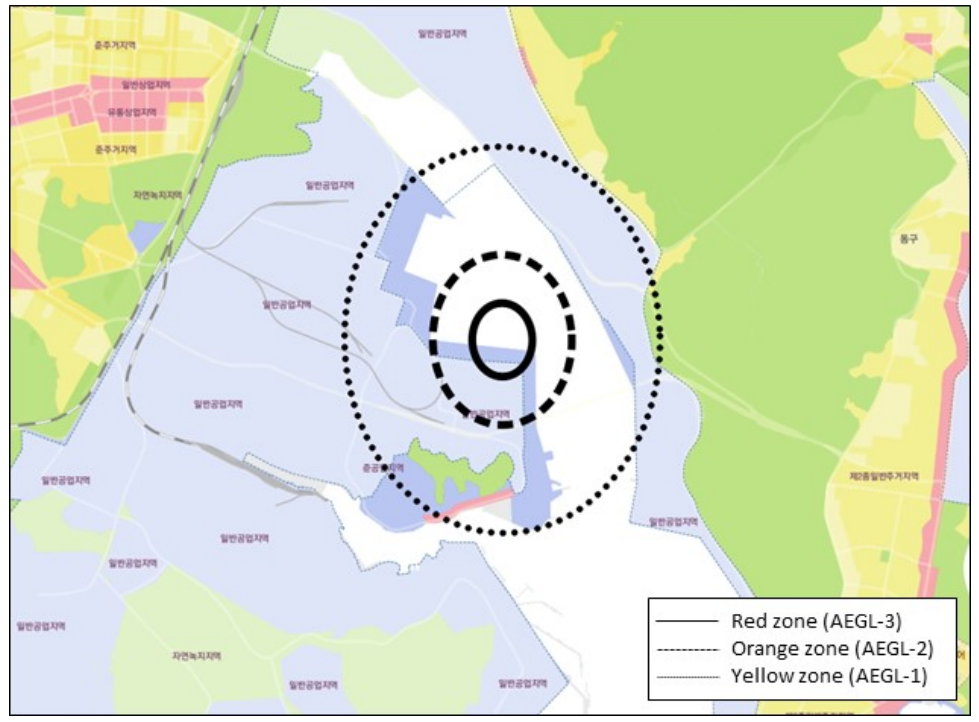

Figure 7: Risk assessment map. 


\section{CONCLUSION}

The accident of Hanyang Ace was evaluated by using HNS dispersion model. In addition, the risk assessment of Ulsan Port was estimated based on the HNS spill scenario. Through the comparison of Hanyang Ace's accident with the numerical model, the simulation results were overestimated. However, the shapes of the crack on the tank were different between actual case and simulation and this led to have overestimation of the values. It is a limitation of the numerical model. This needs to be accounted on the future studies. Furthermore, the HNS spill scenarios of Ulsan Port and risk assessment map were established in this study. Throughout the study results, spilt HNS were only affected to the people who work in industrial area of Ulsan Port. However, the scenarios of HNS spill needed to be account all possible environmental factors to provide more precise result. Therefore, we expect to have more reliable risk assessment by considering the additional types of HNS, weather conditions and HNS spill volume in future studies.

\section{ACKNOWLEDGEMENT}

This research was supported by a grant [MPSS-CG-2015-01] through the Disaster and Safety Management Institute funded by Ministry of Public Safety and Security of Korean government.

\section{REFERENCES}

[1] IMO, Protocol on Preparedness, Response and Co-operation to pollution Incidents by Hazardous and Noxious Substances (OPRC-HNS Protocol). International Maritime Organization, 2000.

[2] Korea Coast Guard, KCG annual accident report, 2016. (in Korean)

[3] Neuparth, T., Moreira, S., Santos, M.M. \& Reis-Henriques, M.A., Hazardous and noxious substances (HNS) in the marine environment: prioritizing HNS that pose major risk in a European context. Marine pollution Bulletin, 62, pp. 21-28, 2011.

[4] Fuhrer, M., Person, O., Hofer, T., Morrissette, M. \& Le Floch, S., Offshore experiments on styrene spillage in marine waters for risk assessment. Marine pollution Bulletin, 64, pp. 1367-1374, 2012.

[5] Garcia, D.A., Cumo, F., Gugliermetti, F. \& Rosa, F., Hazardous and Noxious Substances (HNS) risk assessment along the Italian coastline. Chemical Engineering Transactions, 32, pp. 115-120, 2013.

[6] Lee, M. \& Jung, J., Risk assessment and national measure plan for oil and HNS spill accidents near Korea. Marine pollution Bulletin, 73, pp. 339-344, 2013.

[7] Cho, S., Kim, D. \& Choi, K., Hazardous and Noxious Substances (HNS) risk assessment and accident prevention measures on domestic marine transportation. Journal of the Korean Society of Marine Environment \& Safety, 19(2), pp. 145-154, 2013.

[8] Ulsan Regional Oceans \& Fisheries Administration. A case book of Hanyang Ace, 2015.

[9] Henry, R.E. \& Fauske, H.K., 2-Phase critical flow of one-component mixtures in nozzles orifices and short tubes. Journal of Heat Transfer, 93(2), pp. 179-187, 1971

[10] Fauske, H.K. \& Epstein, M., Source term considerations in connection with chemical accidents and vapor cloud modelling. International Conference on Vapor Cloud Modelling, Cambridge, 1987.

[11] Palazzi, E., Defaveri, M., Fumarola, G. \& Ferraiolo, G., Diffusion from a steady source of short duration. Atmospheric Environment, 16(12), pp. 2785-2790, 1982. 
[12] Hanna, S.R., Gary, A.B. \& Rayford P.H., Handbook on atmospheric diffusion: prepared for the office of Health and Environmental Research, Office of Energy Research, U.S. Department of Energy. Oak Ridge, Tenn: Technical Information Center, U.S. Dept. of Energy, 1982.

[13] Colenbrander, G.W. \& Puttock, J.S., Dense gas dispersion behavior: Experimental observations and model developments. International Symposium on Loss Prevention and Safety Promotion in the Process Industries, Harrogate, England, 1983.

[14] Harold, P.D. Souza, A.S., Louchart P., Russel, D. \& Brunt, H., Development of a riskbased prioritization methodology to inform public health emergency planning and preparedness in case of accidental spill at sea of hazardous and noxious substance (HNS). Environmental International, 72, pp. 157-163. 